\title{
Enhanced tumor response to radiotherapy after PD-1 blockade in metastatic gastric cancer
}

\author{
Akinori Sasaki $^{1,2}$ - Yoshiaki Nakamura ${ }^{1}$. Yosuke Togashi ${ }^{3} \cdot$ Hirofumi Kuno ${ }^{4} \cdot$ Hidehiro Hojo $^{5}$. Shunichiro Kageyama ${ }^{5}$. \\ Naoki Nakamura ${ }^{5} \cdot K_{\text {Kenji Takashima }}{ }^{6} \cdot$ Tomohiro Kadota $^{6} \cdot$ Yusuke Yoda $^{6} \cdot$ Saori Mishima ${ }^{1} \cdot$ Kentaro Sawada $^{1}$. \\ Daisuke Kotani ${ }^{1} \cdot$ Akihito Kawazoe $^{1}$. Yasutoshi Kuboki ${ }^{1} \cdot$ Hiroya Taniguchi ${ }^{1} \cdot$ Takashi Kojima $^{1} \cdot$ Toshihiko Doi $^{1}$. \\ Takayuki Yoshino $^{1} \cdot$ Tomonori Yano $^{6} \cdot$ Tatsushi Kobayashi $^{4} \cdot$ Tetsuo Akimoto $^{2,5} \cdot$ Hiroyoshi Nishikawa $^{3} \cdot$ Kohei Shitara $^{1}$
}

Received: 10 January 2020 / Accepted: 6 March 2020 / Published online: 16 March 2020

(c) The International Gastric Cancer Association and The Japanese Gastric Cancer Association 2020

\begin{abstract}
Background Immune checkpoint inhibitors may enhance the efficacy of radiotherapy (RT) in cancer treatment but the effect remains unknown in metastatic gastric cancer (mGC). This study aimed to compare the tumor shrinkage by palliative RT for $\mathrm{mGC}$ patients with or without previous exposure to anti-PD-1 therapy.

Methods Data of 36 mGC patients who had received palliative RT from April 2013 to May 2019 were analyzed. Primary tumor responses were evaluated through a volumetric measurement-based method using computed tomography (CT) and endoscopic responses were evaluated in patients who underwent endoscopy before and after RT. Tumor microenvironment (TME) immune status was investigated by analyzing tumor-infiltrating lymphocytes by flow cytometry.

Results Among 36 patients, 18 had previous exposure to anti-PD-1 before RT showing no significant differences in baseline characteristics with the other 18 patients without exposure to anti-PD- 1 treatment. Tumor responses were observed in $28 \%$ $(5 / 18)$ and none $(0 / 18)$ in the anti-PD-1-exposed vs. naïve group, respectively $(P=0.045)$. Five out of eight patients in the anti-PD-1-exposed group, who underwent endoscopy after RT showed partial response, but none in the anti-PD-1-naïve patients showed response $(P=0.026)$. Increase in the $\mathrm{CD}^{+} \mathrm{T}$ cell/effector regulatory $\mathrm{T}$ cell ratio in TILs after anti-PD-1 therapy was noted in three responders to RT, but not in the other three non-responders.

Conclusions Prior exposure to anti-PD-1 therapy increases tumor response to RT. Immune profiling suggests that anti-PD-1 therapy may enhance the efficacy of RT by immunoactivation in the TME.

Keywords Radiotherapy · Immune checkpoint inhibitors · Anti-PD-1 therapy · Tumor-infiltrating lymphocytes

\section{Abbreviations}

PD-1 Programmed death-1

RT Radiotherapy

mCG Metastatic gastric cancer

TME Tumor microenvironment

TIL Tumor infiltrating lymphocyte

ICI Immune checkpoint inhibitor

Akinori Sasaki, Yoshiaki Nakamura, and Yosuke Togashi contributed equally to this work.

Electronic supplementary material The online version of this article (https://doi.org/10.1007/s10120-020-01058-4) contains supplementary material, which is available to authorized users.

Kohei Shitara

kshitara@east.ncc.go.jp

Extended author information available on the last page of the article

PD-L1 Programmed death-ligand 1

PFS Progression-free survival

OS Overall survival

PFS Progression-free survival

ECOG PS Eastern Cooperative Oncology Group performance status

HER2 Human epidermal growth factor receptor 2

MMR Mismatch repair

EBV Epstein-Barr virus

IHC Immunohistochemistry

FISH Fluorescence in situ hybridization

TC Tumor cell

IC Immune cell

CPS Combined positive score

MLH1 Anti-mutL homolog 1

MSH2 Anti-mutS homolog 2

PMS2 Anti-postmeiotic segregation increased 2
\end{abstract}




$\begin{array}{ll}\text { MSH6 } & \text { Anti-mutS homolog 6 } \\ \text { EBER } & \text { EBV-encoded RNA } \\ \text { TMB } & \text { Tumor mutation burden } \\ \text { mt/MB } & \text { Mutations/megabase } \\ \text { HR } & \text { Hazard ratio } \\ \text { CI } & \text { Confidence interval } \\ \text { PR } & \text { Partial response } \\ \text { SD } & \text { Stable disease } \\ \text { PD } & \text { Progressive disease } \\ \text { FOXP3 } & \text { Forkhead box protein P3 } \\ \text { PBS } & \text { Phosphate-buffered saline } \\ \text { IRF3 } & \text { Interferon regulatory factor 3 } \\ \text { IFN } & \text { Type I interferon }\end{array}$

\section{Introduction}

Systemic chemotherapy is the standard treatment option for patients with metastatic gastric cancer (mGC). However, it is usually inadequate for palliation of local symptoms, often resulting in the need for additional interventions. Several reports have suggested that palliative radiotherapy (RT) is effective in relieving tumor pain, bleeding, and obstruction caused by the primary tumor in patients with mGC, and it has been recognized as a viable, non-invasive, palliative therapeutic option [1-3].

Recently, immune checkpoint inhibitors (ICIs) have been reported to demonstrate anti-tumor immune responses by activating effector $\mathrm{T}$ cells in various types of cancers [4]. Nivolumab, a monoclonal antibody against programmed cell death protein-1 (PD-1), showed survival benefit for patients with $\mathrm{mGC}$ that had progressed despite two or more previous chemotherapy regimens [5]. However, the objective response rate was only $11 \%$, necessitating the need for more effective therapies to achieve tumor shrinkage.

As preclinical models have shown, the combination of RT and immunotherapy may be more potent than lone treatment with either of the two options [6-8]. Several case reports have showed that the combination of radiation and immunotherapy is beneficial for treating different cancer types [9-13]. A recent phase III trial (PACIFIC) in which programmed cell death-ligand 1 (PD-L1) antibody durvalumab was administered as consolidation therapy after chemoradiotherapy for stage III non-small cell lung cancer showed substantial improvement in progression-free survival (PFS), thus suggesting synergism between RT and anti-PD-1 therapy $[14,15]$. However, the effect of anti-PD-1 therapy on the therapeutic efficacy of RT in mGC patients remains unclear. In this study, we assessed tumor shrinkage achieved by palliative RT in patients with mGC who had prior exposure to anti-PD-1 therapy in comparison with patients without prior exposure. In addition, we examined the immune status of the tumor microenvironment (TME) in $\mathrm{mGC}$ patients to elucidate the potential role of prior anti-PD-1 therapy in enhancing RT outcomes.

\section{Methods}

\section{Patients}

A retrospective study was performed to examine tumor shrinkage after palliative RT in patients with mGC (RT cohort). The inclusion criteria for this study were: (1) presence of histologically proven gastric adenocarcinoma; (2) history of palliative RT for bleeding, tumor pain, and tumor obstruction caused by the primary tumor at a dose of $30 \mathrm{~Gy}$ in ten fractions from April 2013 to February 2019 at the National Cancer Center Hospital East; and (3) availability of computed tomography (CT) scans before initiation of RT, and within a month after completion of RT. Patients who received chemotherapy within the period between $\mathrm{CT}$ evaluation, and initiation or completion of RT were excluded. Patients were divided into two groups based on prior exposure to anti-PD-1 therapy: anti-PD-1-exposed and anti-PD1-naïve groups. Furthermore, in order to compare between the TME immune status following anti-PD-1 therapy or cytotoxic chemotherapy, mGC patients with progressive disease (PD) who had received anti-PD-1 therapy or fluoropyrimidine plus oxaliplatin, and had available paired biopsy samples before and after treatment were included in another cohort. All patients provided written informed consent prior to radiotherapy. Further, patients who underwent biomarker analysis provided written informed consent for the analysis. The study protocol of biomarker research was approved by the Institutional Review Board at the National Cancer Center Japan.

\section{Assessment}

The primary objective of the study was to investigate the response of the primary tumor to RT after prior anti-PD-1 therapy. Since the response of primary tumor in GCs is difficult to measure by the Response Evaluation Criteria in Solid Tumors version (RECIST) approach [16], a volumetric measurement-based method was used to evaluate the response of primary lesions in this study. In this method, the volumes of the lesions were calculated by summing each of the 2D volumes of the entire lesion. The percentage volume reduction was calculated using the following equation: [(pre-radiotherapy value) - (post-radiotherapy value $)] /($ pre-radiotherapy value $) \times 100$. A previous study demonstrated that a $64.5 \%$ reduction in tumor size assessed using CT scans with the volumetric measurement-based method described above corresponded to histopathologic regression in gastric cancers treated with neoadjuvant 
chemotherapy [17]. Based on this, for the current study, tumor response was defined as having more than a $70 \%$ reduction in tumor volume. Segmentation of the whole primary lesion and calculation of the percentage volume reduction were independently performed by two different radiologists (HK and KK) who were blinded to the clinical findings. They used 3D volumetric analysis with OsiriX Imaging Software (Pixmeo, Geneva, Switzerland) (Additional File: Fig. S1) to calculate the percentage volume reduction. Endoscopic responses were evaluated in patients who underwent endoscopy after completion of RT by three endoscopists (KT, TK, and YY). The primary lesion response to treatment consisted of endoscopic complete response (eCR) and endoscopic partial response (ePR). Endoscopic complete response (eCR) was defined as disappearance of all tumors, while endoscopic partial response (ePR) was defined as tumor decrease by more than two-thirds of the major axis, one-half of the area, or one-third of the volume based on the Japanese Classification of Gastric Carcinoma, 15th Edition [18]. Tumor responses in metastatic sites were assessed according to the RECIST v1.1. Adverse events (AEs) were monitored during and after RT and were graded according to the Common Terminology Criteria for Adverse Events version 5.0.

Molecular characteristics, such as the status of human epidermal growth factor receptor 2 (HER2), PD-L1, mismatch repair (MMR), Epstein-Barr virus (EBV), and genetic alterations, were analyzed with formalin-fixed, paraffin-embedded tissue specimens from archival tissue samples where available (Additional File: Tables S1 and S2).

Fig. 1 CONSORT flow diagram

\section{Tumor infiltrating lymphocyte (TIL) analysis}

To investigate the immune profile of fresh biopsy samples of patients, particularly the activity of regulatory $\mathrm{T}$ (Treg) cells, we used flow-cytometry for TIL analysis. To collect TILs, tumor tissues were minced and treated with gentleMACS ${ }^{\text {TM }}$ Dissociator (Miltenyi Biotec, Bergisch Gladbach, North Rhine-Westphalia, Germany), and the collected TILs were subjected to flow-cytometry as earlier stated [19]. Identification of $\mathrm{CD}^{+}{ }^{+}$Treg cells in humans is compromised due to the upregulation of Forkhead box protein P3 (FOXP3) upon T cell receptor stimulation by conventional $\mathrm{T}$ cells [20]. We therefore proposed a classification of human Treg cells based on the expression levels of a naïve marker CD45RA and that of FOXP3 $[21,22]$. FOXP $^{+} \mathrm{CD}^{+} \mathrm{T}$ cells can thus be divided into three fractions namely: naïve Treg cells $\left(\mathrm{CD} 45 \mathrm{RA}^{+} \mathrm{FOXP} 3^{\text {low }} \mathrm{CD} 4^{+}\right.$): (I), effector Treg (eTreg) cells $\left(\mathrm{CD} 45 \mathrm{RA}^{-} \mathrm{FOXP} 3^{\text {high }} \mathrm{CD} 4^{+}\right)$: (II), and non-Treg cells $\left(\mathrm{CD} 45 \mathrm{RA}^{-} \mathrm{FOXP} 3^{\text {low }} \mathrm{CD}^{+}\right)$): (III). Although eTreg cells are difficult to identify by immunohistochemistry, they harbor strong immunosuppressive functions.

\section{Flow-cytometry}

Cells were washed with a solution of phosphate-buffered saline (PBS) and 2\% fetal calf serum and were subsequently stained with monoclonal antibodies specific for CD3, CD4, CD8, and CD45RA, and a fixable viability dye. After staining of cell surface markers, cells were intracellularly stained for FOXP3 with FOXP3 Staining Buffer Set (Thermo Fisher Scientific) according to the manufacturer's

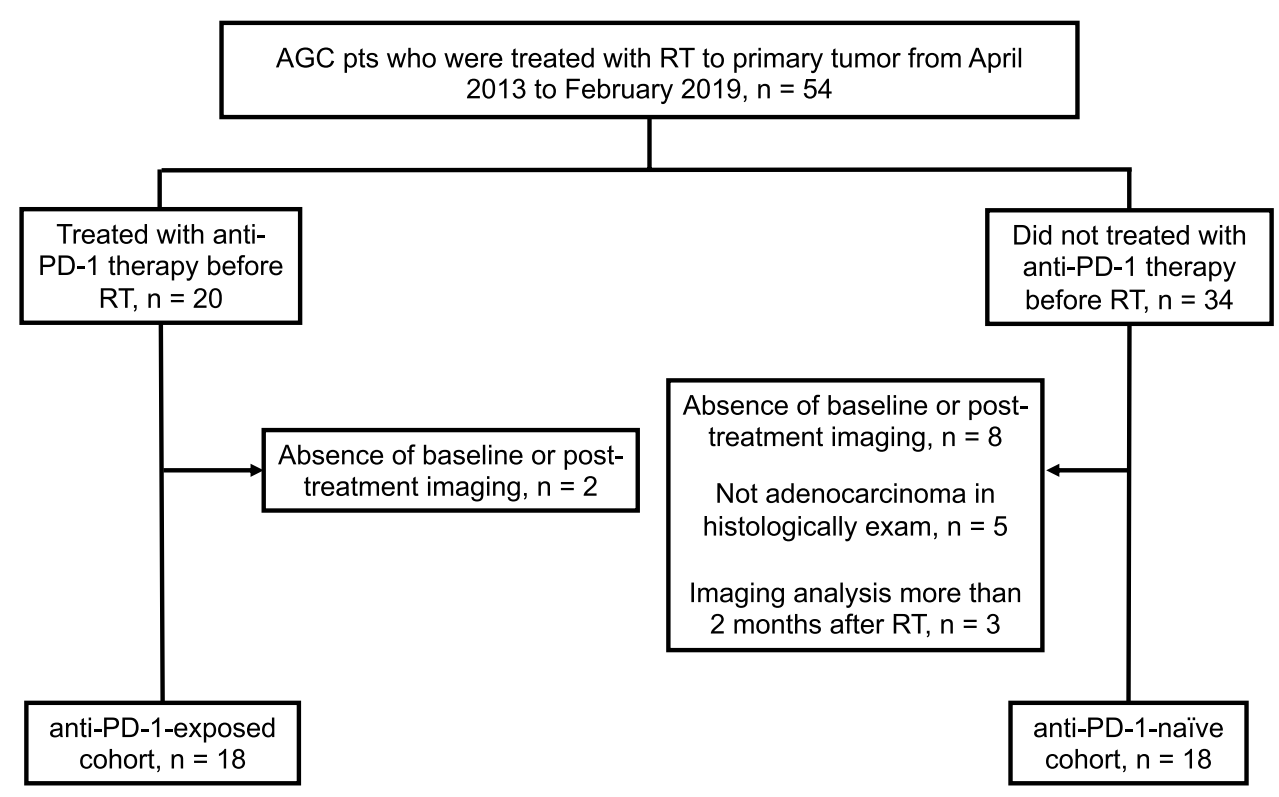


instructions. After staining, cells were analyzed with BD LSRFortessa $^{\mathrm{TM}}$ X-20 (BD Biosciences) and FlowJo software (BD Biosciences). Staining antibodies were diluted according to the manufacturer's instructions. Detailed information on the antibodies used is summarized in Table S3.

\section{Statistical analysis}

Statistical comparisons of baseline characteristics between anti-PD-1-exposed and anti-PD-1-naive patients were performed using $\chi^{2}$ or Fisher's exact test for categorical data and Student's $t$ test or Mann-Whitney's test for continuous data. All statistical analyses were performed with 5\% alpha risk or $95 \%$ confidence intervals using SPSS version 25 (IBM, Chicago, IL).

\section{Results}

\section{RT cohort patient characteristics}

Among 54 patients with mGC who received RT for primary lesion during the study period, 18 were excluded because of the absence of baseline or post-treatment imaging, no adenocarcinoma in histologically examination and imaging analysis more than 2 months after RT. Thus, a total of 36 patients were eligible for inclusion in this cohort, and 18 of them had received anti-PD-1 therapy before RT (Fig. 1). Table 1 shows patient characteristics. All patients in the anti-PD-1-exposed group and nine out of 18 patients in the anti-PD-1-naïve group had received more than three chemotherapeutic regimens before RT $(P=0.001)$. Other clinicopathological baseline characteristics were not significantly different between the two groups. While molecular characteristics, including HER2, MMR, EBV, PD-L1 status, and genetic alterations, were available for a limited number patients due to the lack of archival samples, there were no significant differences between the groups, except for TP53 mutation status ( $78 \%$ vs. $25 \%, P=0.03$ ) (Table S4).

\section{Tumor response to RT}

CT showed that five patients achieved tumor response in the anti-PD-1-exposed group, while no patient achieved response in the anti-PD-1-naïve group (28\% vs. $0 \%$, $P=0.045$ ) (Fig. 2). No significant differences were observed in the proportion of patients who achieved palliation of symptoms between the anti-PD-1-exposed and naïve groups (77.8\% vs. $66.7 \%, P=0.71$ ). In the anti-PD-1 exposed group, there were no significant differences in clinicopathologic and molecular characteristics, including histological type,
Table 1 Patients' characteristics in RT cohort

\begin{tabular}{|c|c|c|c|c|}
\hline Features & Available & $\begin{array}{l}\text { Anti-PD- } \\
1 \text {-exposed } \\
\text { group } \\
(n=18)\end{array}$ & $\begin{array}{l}\text { Anti-PD- } \\
\text { 1-naïve } \\
\text { group } \\
(n=18)\end{array}$ & $P$ value \\
\hline Age, $\geq 65, n(\%)$ & & $10(55.6)$ & $13(72.2)$ & 0.49 \\
\hline Male, $n(\%)$ & & $16(88.9)$ & $16(88.9)$ & 1.00 \\
\hline \multicolumn{5}{|l|}{ ECOG PS, $n(\%)$} \\
\hline 0 or 1 & & $14(77.8)$ & $16(88.9)$ & 0.66 \\
\hline 2 & & $4(22.2)$ & $2(11.1)$ & \\
\hline \multicolumn{5}{|c|}{ Reason for RT procedure } \\
\hline Bleeding & & $13(72.2)$ & $15(83.3)$ & 0.67 \\
\hline Obstruction & & $4(22.2)$ & $2(11.1)$ & \\
\hline Pain & & $1(5.6)$ & $1(5.6)$ & \\
\hline \multicolumn{5}{|l|}{ Histology, $n(\%)$} \\
\hline Intestinal & & $11(61.1)$ & $14(77.8)$ & 0.47 \\
\hline Diffuse & & 7 (38.9) & $4(22.2)$ & \\
\hline \multicolumn{5}{|c|}{ Previous treatment regimens, $n(\%)$} \\
\hline$\leq 2$ & & $0(0.0)$ & $9(50.0)$ & 0.001 \\
\hline$\geq 3$ & & $100(100.0)$ & $9(50.0)$ & \\
\hline \multicolumn{5}{|c|}{ Organs with metastases, $n(\%)$} \\
\hline$\leq 2$ & & $11(61.1)$ & $8(44.4)$ & 0.51 \\
\hline$\geq 3$ & & $7(38.9)$ & $10(55.6)$ & \\
\hline HER2, $n(\%)$ & 36 & & & \\
\hline Negative & & $16(88.9)$ & $14(77.8)$ & 0.66 \\
\hline Positive & & $2(11.1)$ & $4(22.2)$ & \\
\hline MMR, $n(\%)$ & 27 & & & \\
\hline Proficient & & $13(76.5)$ & $6(60.0)$ & 0.42 \\
\hline Deficient & & $4(23.5)$ & $4(40.0)$ & \\
\hline $\mathrm{EBV}, n(\%)$ & 28 & & & \\
\hline Negative & & $17(100.0)$ & $11(100.0)$ & - \\
\hline Positive & & $0(0.0)$ & $0(0.0)$ & \\
\hline PD-L1 CPS, $n(\%)$ & 27 & & & \\
\hline$<1$ & & $4(23.5)$ & $0(0.0)$ & 0.26 \\
\hline$\geq 1$ & & $13(76.5)$ & $10(100.0)$ & \\
\hline PD-L1 CPS, $n(\%)$ & 27 & & & \\
\hline$<10$ & & $15(88.2)$ & $8(80.0)$ & 0.61 \\
\hline$\geq 10$ & & $2(11.8)$ & $2(20.0)$ & \\
\hline
\end{tabular}

$P D-1$ programmed cell death protein-1, $R T$ radiotherapy, ECOG $P S$ Eastern Cooperative Oncology Group performance status, HER2 human epidermal growth factor receptor related 2, MMR mismatch repair, $E B V$ Epstein-Barr virus, $P D-L 1$ programmed death-ligand 1, $T C$ tumor cell, $C P S$ combined positive score

response to anti-PD-1 therapy, number of infusions of the anti-PD-1 antibody, and PD-L1 status, between patients who showed response to RT and those who did not (Table 2). The intervals from the last dose of anti-PD-1 therapy were not significantly different (15 [2-148] vs. 32 [1-180] days, $P=0.64)$.

Fifteen patients underwent endoscopic examination both before and after RT. In the anti-PD-1-exposed group, 
Fig. 2 Waterfall plot of patients in both anti-PD-1-exposed and anti-PD-1-naïve cohort

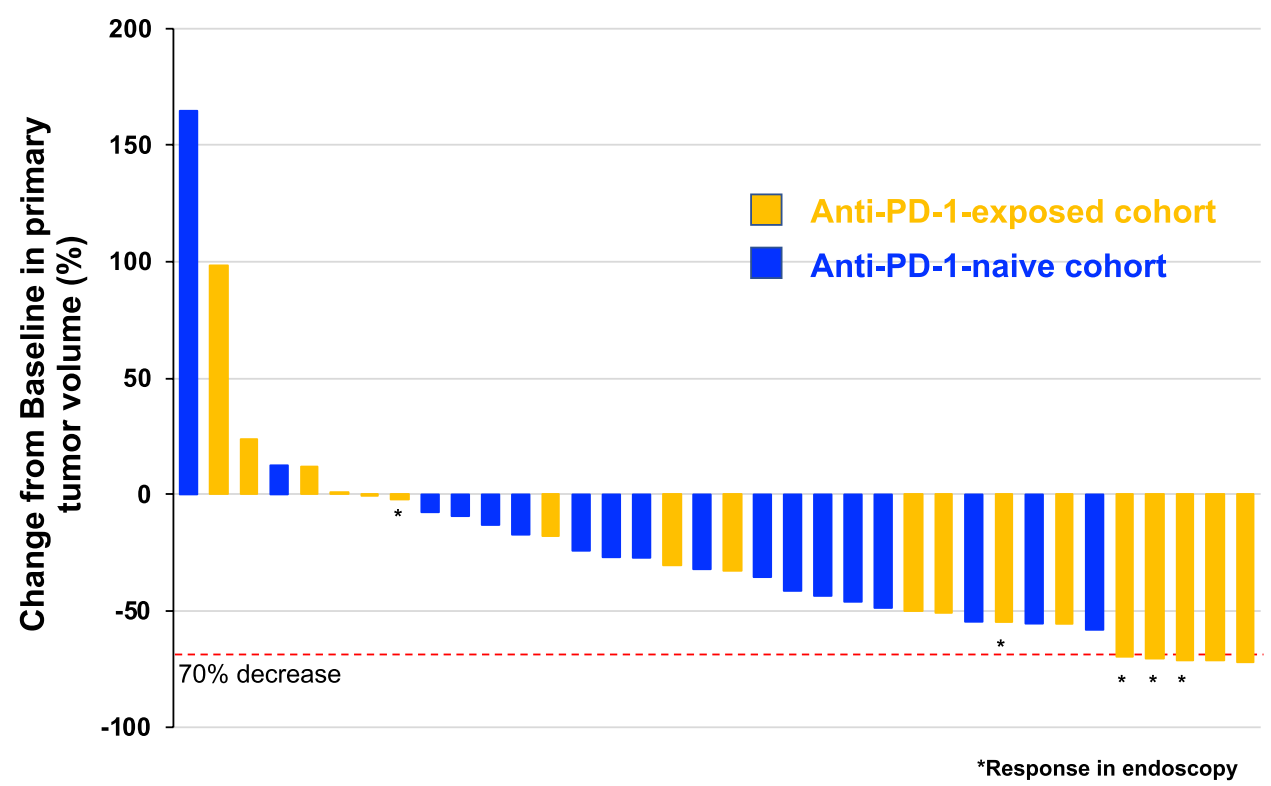

five patients, including three patients with tumor response based on CT findings, achieved endoscopic response, while no patient had endoscopic response in the anti-PD-1-naïve group (63\% vs. $0 \%, P=0.026$ ) (Figs. 2, 3a, b). No patient in either the anti-PD-1-exposed or anti-PD-1-naïve groups achieved complete response or partial response in distant metastases as an abscopal effect (Fig. S2).

There were no significant differences in the incidence of AEs between anti-PD-1-exposed and anti-PD-1-naïve group (Table 3). The most common AE was nausea. All adverse events were transient and resolved after completion of RT. In addition, no patients developed immune-related AEs during and after RT in anti-PD-1-exposed patients.

\section{TIL analysis}

The TILs were analyzed in six anti-PD-1 exposed patients in the RT cohort. TIL analysis showed that the frequency of $\mathrm{CD}^{+} \mathrm{T}$ cells was increased and that of eTreg cells was decreased after administration of an anti-PD-1 antibody in three patients who achieved tumor response to RT (Fig. 4a). However, no such change was observed in the other three patients who had no tumor response to RT.

In addition, to elucidate the changes in the TME immune status caused by anti-PD-1 treatment, we analyzed additional TILs from 15 patients who received an anti-PD-1 antibody (Additional File: Table S5). Data from these patients were combined with data collected from the six patients in the RT cohort. In patients who received anti-PD-1 therapy there was a significant increase in the $\mathrm{CD}^{+} \mathrm{T}$ cell/eTreg cell ratio (Fig. 4b) resulting from a significant increase in the frequency of $\mathrm{CD}^{+} \mathrm{T}$ cells and a significant decrease in the frequency of eTreg cells in TILs following treatment, even at the PD state. To compare the changes in the TME immune status between anti-PD-1 treatment and cytotoxic chemotherapy, we analyzed TILs from ten patients who received fluoropyrimidine plus oxaliplatin treatment (Additional File: Table S5). All TILs were analyzed using paired primary tumor samples collected both before treatment initiation and after completion. Treatment duration in the group that received anti-PD-1 was significantly shorter than that in the group that received cytotoxic chemotherapy (median 1.2 months vs. 3.3 months, $P=0.022$ ). Observations made for the anti-PD-1 exposed patients were not found in patients who received fluoropyrimidine plus oxaliplatin (Fig. 4c). These findings suggest that anti-PD-1 therapy may improve the TME immune status even at PD state, a phenomenon not associated with cytotoxic chemotherapy.

\section{Discussion}

In this study, we evaluated the efficacy of RT in patients with $\mathrm{mGC}$ who received anti-PD-1 therapy prior to RT. CT findings showed that $28 \%$ of $\mathrm{mGC}$ patients who received RT after prior anti-PD-1 therapy showed tumor response, while patients who received no prior anti-PD-1 therapy had no response to RT. In addition, endoscopic responses were observed in $63 \%$ of anti-PD-1-exposed patients while no patient in the anti-PD-1-naïve group showed endoscopic response. These findings suggest that anti-PD-1 therapy potentially enhances the sensitivity of mGC to RT. To the best of our knowledge, this is the first study 
Table 2 Characteristics of responders and non-responders in Anti-PD-1-exposed group in RT cohort

\begin{tabular}{|c|c|c|c|c|}
\hline Features & Available & $\begin{array}{l}\text { Response by CT } \\
\text { scan }(n=5)\end{array}$ & $\begin{array}{l}\text { No response by CT } \\
\text { scan }(n=13)\end{array}$ & $P$ value \\
\hline Age, $\geq 65, n(\%)$ & & $4(80.0)$ & $6(46.2)$ & 0.31 \\
\hline Male, $n(\%)$ & & $5(100.0)$ & $11(84.6)$ & 1.00 \\
\hline \multicolumn{5}{|l|}{ ECOG PS, $n(\%)$} \\
\hline 0 or 1 & & $3(60.0)$ & $11(84.6)$ & 0.53 \\
\hline 2 & & $2(40.0)$ & $2(14.4)$ & \\
\hline \multicolumn{5}{|l|}{ Histology, $n(\%)$} \\
\hline Intestinal & & $2(40.0)$ & $9(69.2)$ & 0.33 \\
\hline Diffuse & & $3(60.0)$ & $4(30.8)$ & \\
\hline \multicolumn{5}{|l|}{ Previous gastrectomy, $n(\%)$} \\
\hline No & & $5(100.0)$ & $11(84.6)$ & 1.00 \\
\hline Yes & & $0(0.0)$ & $2(15.4)$ & \\
\hline \multicolumn{5}{|l|}{ Organs with metastases, $n(\%)$} \\
\hline$\leq 2$ & & $3(60.0)$ & $8(61.5)$ & 1.00 \\
\hline$\geq 3$ & & $2(40.0)$ & $5(38.5)$ & \\
\hline PD-L1, $n(\%)$ & 17 & & & \\
\hline Positive in TC & & $2(50.0)$ & $1(7.7)$ & 0.12 \\
\hline Positive in IC & & $4(100.0)$ & $9(69.2)$ & 0.52 \\
\hline Any expression & & $4(100.0)$ & $9(69.2)$ & 0.52 \\
\hline No expression & & $0(0.0)$ & $4(30.8)$ & \\
\hline PD-L1 CPS, $n(\%)$ & 17 & & & \\
\hline$<1$ & & $0(0.0)$ & $4(30.8)$ & 0.52 \\
\hline$\geq 1$ & & $4(100.0)$ & $9(69.2)$ & \\
\hline PD-L1 CPS, $n(\%)$ & 17 & & & \\
\hline$<10$ & & $3(75.0)$ & $12(92.3)$ & 0.43 \\
\hline$\geq 10$ & & $1(25.0)$ & $1(7.7)$ & \\
\hline \multicolumn{5}{|l|}{ Response to anti-PD-1 } \\
\hline PR & & $0(0.0)$ & $3(23.1)$ & 0.47 \\
\hline SD & & $3(60.0)$ & $5(38.5)$ & \\
\hline PD & & $2(40.0)$ & $5(38.5)$ & \\
\hline $\begin{array}{l}\text { Interval from last dose of anti-PD-1, days } \\
\text { Median (range) }\end{array}$ & & $15(2-148)$ & $32(1-180)$ & 0.64 \\
\hline Number of Anti-PD-1 infusion, median & & $6(1-8)$ & $5(1-11)$ & 1.00 \\
\hline
\end{tabular}

$P D$-1 programmed cell death protein-1, ECOG PS Eastern Cooperative Oncology Group performance status, $P D$ - $L 1$ programmed death-ligand 1, TC tumor cell, $C P S$ combined positive score, $P R$ partial response, $S D$ stable disease, $P D$ progressive disease to report a dramatic response to RT after prior anti-PD-1 therapy in patients with $\mathrm{mGC}$.

Although the efficacy of the combination of ICIs and RT has been reported in some studies where RT is administered before ICIs or concurrently with ICIs, some preclinical and clinical studies have demonstrated the radiosensitizing effect of ICIs [23-25]. Retrospective studies reported that patients who received ipilimumab before RT had increased duration of irradiated tumor response compared to patients who received ipilimumab after RT [24, 26]. Similarly, our study demonstrated the radiosensitizing effect of anti-PD-1 therapy in patients with mGC. The clinicopathologic and molecular characteristics, including the efficacy of prior-anti-PD-1 therapy and ICI-related biomarkers such as PD-L1 status, were not significantly different between patients who showed response to RT after prior anti-PD-1 therapy and those who did not. This suggests that the response can be explained, not only by the direct effect of RT, but also by the radiosensitizing effect of anti-PD-1 therapy. Interestingly, responses to RT were observed even up to 148 days after the last dose of anti-PD-1 therapy. This is consistent with the findings of a previous study, which showed that the PD-1 blocking effect of anti-PD-1 antibody persisted in patients for more than 20 weeks after the last infusion [27].

It has been known that RT induces cancer cell DNA damage promoting apoptosis and subsequent reproductive death [28]. However, recent evidence suggests that 


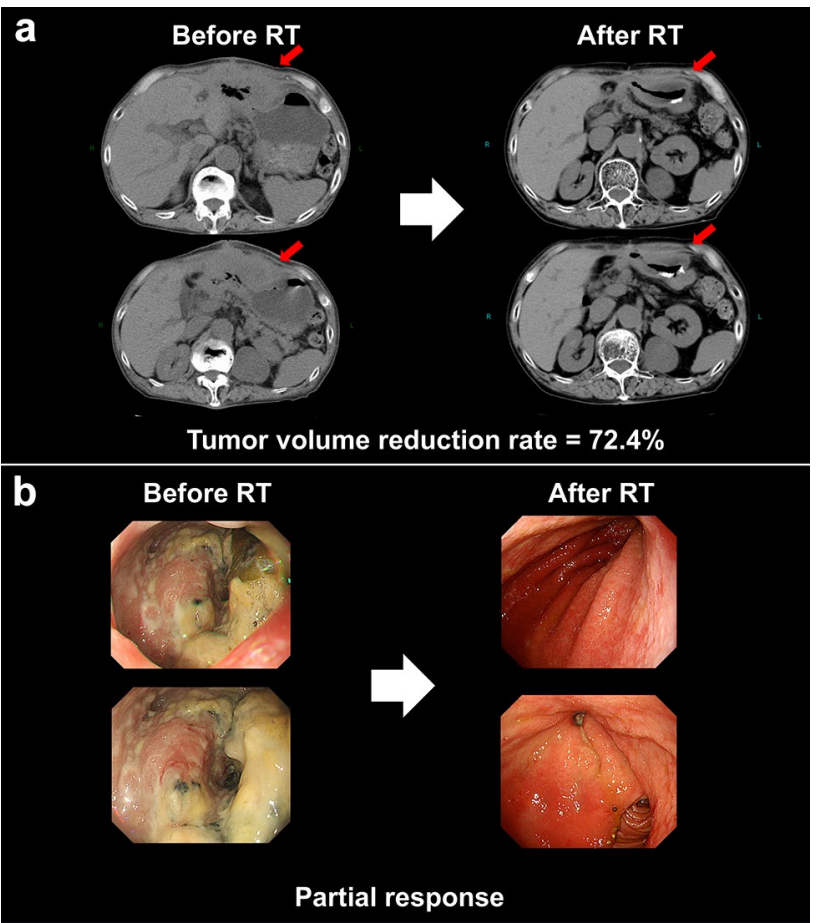

Fig. 3 Representative images from the patient with treatment response to RT. Patient G717 was treated with six cycles of nivolumab for stable disease and then received RT for the primary tumor to palliate the symptom of anemia. After RT, the tumor elicited response on both CT scan (a) and endoscopic examination (b), and the patient was relieved of the symptom of anemia

RT induces cell death via activation of the immune system as well as direct DNA damage [29]. Activation of the cGAS-STING pathway is one of the most crucial role in the immune system, although several other mechanisms have been suggested. The DNA damage caused by RT activates the cGAS-STING pathway, which in turn activates interferon regulatory factor 3 (IRF3) to induce type I interferon (IFN) production [30]. This RT induced type I IFN signaling in dendritic cells promotes the crosspriming of $\mathrm{CD}^{+}{ }^{+} \mathrm{T}$ cells, leading to $\mathrm{CD} 8^{+}$effector $\mathrm{T}$ cell activation and subsequent tumor regression. In fact, some studies have reported that an increased number of $\mathrm{CD} 8^{+}$ $\mathrm{T}$ cells independently predicted a better response to RT in cancer patients, suggesting that anti-tumor immunity may play an important role in tumor regression by RT $[31,32]$. Treg cells are an immunosuppressive subset of $\mathrm{CD} 4^{+} \mathrm{T}$ cells, characterized by specific expression of the transcription factor, FOXP3. They are abundant in tumor tissues and play key roles in hindering effective antitumor immunity in cancer patients [33]. Accordingly, an increase in the number of Treg cells is reportedly a poor prognostic factor in cancer patients who receive RT [34]. In our study, the number of $\mathrm{CD} 8^{+} \mathrm{T}$ cells increased and that of eTreg cells decreased in TILs even at PD state for most patients who received anti-PD-1 therapy in RT cohort, resulting in an increased $\mathrm{CD} 8^{+} / \mathrm{eTreg}$ cell ratio. This indicates that anti-tumor immunity in the TME is activated by anti-PD-1 therapy regardless of its efficacy in improving tumor response to RT. In addition, a similar immunologic change was observed in the TME of a patient who responded to RT after prior anti-PD-1 therapy, but this change was not found in patients who did not respond to RT. In sharp contrast, there was no such tendency in patients who received fluoropyrimidine plus oxaliplatin, indicating that the activation of anti-tumor immunity by cytotoxic chemotherapies may be weak compared to antiPD-1 therapy. Altogether, activation of anti-tumor immunity by anti-PD-1 therapy can lead to a dramatic response to $\mathrm{RT}$ via immunological mechanisms.

It is important to note the limitations of the present study. First, this was a retrospective study of patient data from a single institution with a limited sample size. Molecular characteristics, including MMR and PD-L1 status related to ICI responses, were available in a limited number of patients due to the lack of archival samples. Associations between the efficacy of combined radiotherapy and immunotherapy biomarkers should be evaluated in a future study. Endoscopic findings were also available in a limited number of patients since endoscopic examinations were not conducted in some patients due to their general status or symptoms. Furthermore, while we analyzed TILs, the TIL analysis was
Table 3 Adverse events associated with RT

\begin{tabular}{llllll}
\hline & \multicolumn{2}{l}{$\begin{array}{l}\text { Anti-PD-1-exposed group } \\
(n=18)\end{array}$} & & \multicolumn{2}{l}{$\begin{array}{l}\text { Anti-PD-1-naïve group } \\
(n=18)\end{array}$} \\
\cline { 2 - 3 } & All Grade, $n(\%)$ & $\begin{array}{l}\text { Grade 3 or 4, } \\
n(\%)\end{array}$ & & All Grade, $n(\%)$ & $\begin{array}{l}\text { Grade 3 } \\
\text { or 4, } n \\
(\%)\end{array}$ \\
\hline Fatigue & $1(5.6)$ & $0(0.0)$ & & $0(0.0)$ & $0(0.0)$ \\
Decreased appetite & $1(5.6)$ & $0(0.0)$ & & $1(5.6)$ & $0(0.0)$ \\
Nausea & $5(27.8)$ & $0(0.0)$ & & $3(16.7)$ & $0(0.0)$ \\
Dermatitis & $0(0.0)$ & $0(0.0)$ & $1(5.6)$ & $0(0.0)$ \\
\hline
\end{tabular}

$P D-1$ programmed cell death protein-1 

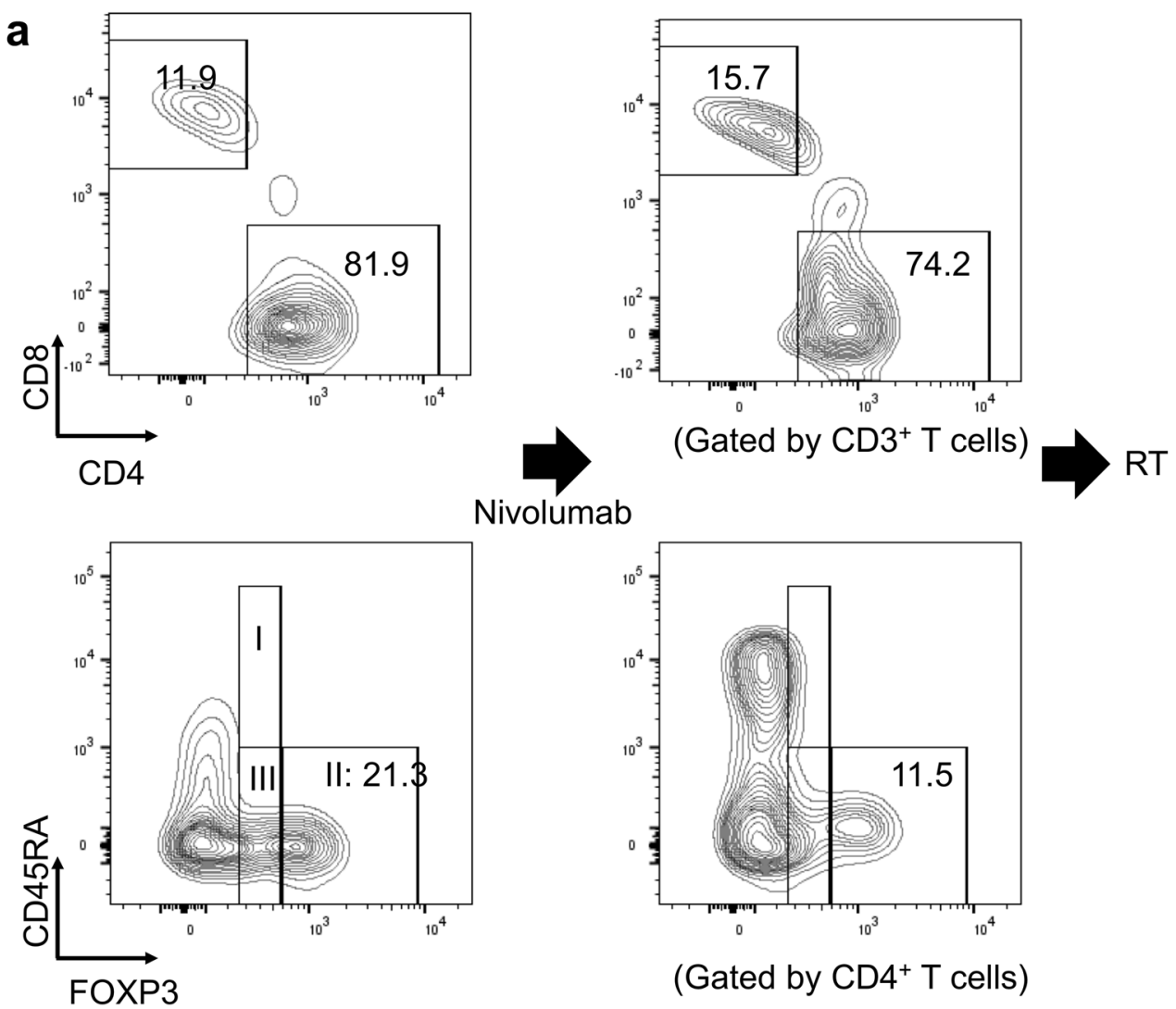

b

Nivolumab

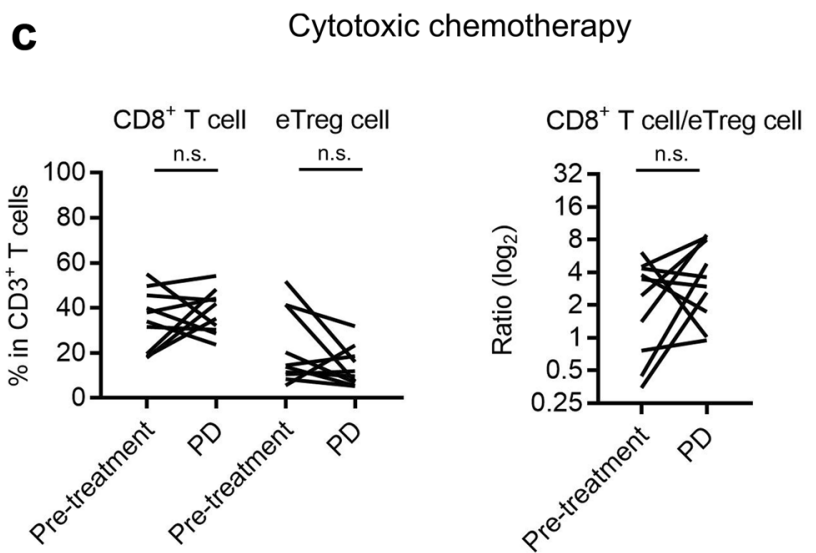

Fig. 4 Tumor infiltrating lymphocyte (TIL) analyses. a Representative staining figures in anti-PD-1-exposed patient with response to RT. Tumor tissues were minced and subsequently analyzed with flow cytometry. RT, radiotherapy (b) and c Kinetic change of $\mathrm{CD} 8^{+} \mathrm{T}$

conducted using a small cohort. Although biopsy samples were generally collected from the site where the tumor surface components were expected to be exposed, results from the analysis of the tumor tissues should be carefully interpreted due to possibilities of intra-tumoral heterogeneity. Therefore, our data may be insufficient to establish the

cells, eTreg cells, and $\mathrm{CD} 8^{+} \mathrm{T}$ cell/eTreg cell ratio in TILs after antiPD-1 (b) versus cytotoxic chemotherapy (c). TILs were analyzed as described in a. *, $P<0.05$; n.s. not significant

mechanism of synergism associated with the combination of radiotherapy and immunotherapy. Due to these limitations, the current study only generates a hypothesis. Therefore, the efficacy and synergistic mechanisms of the combination of radiotherapy and immunotherapy in $\mathrm{mGC}$ patients need to be investigated further. 


\section{Conclusions}

Although, a small sample size is the major limitation of our study, our findings suggested that anti-PD-1 therapy might enhance the sensitivity of primary GC to RT by immunoactivation. These findings merit further investigation in a larger cohort to validate the efficacy of this therapeutic approach.

Acknowledgements We thank Ms. Y. Tada, T. Takaku, M. Nakai, K. Onagawa, M. Takemura, M. Hoshino, K. Yoshida, Y. Osada, and M. Ozawa for their technical assistance.

Author contribution AS, YN, YT, HK, SK and KS designed the study, collected data, performed data analysis and wrote manuscript. NN, KT, TK, YY, SM, KS, DK, AK, YK, HT, TK, TD, TY, TY, TK, TA and $\mathrm{HN}$ were involved in data interpretation and critically reviewing the manuscript. All authors read and approved the final manuscript.

Funding This study was supported by a research funding from National Cancer Center Hospital East (none apply).

Availability of data and materials All data analyzed during this study has been included within the article.

\section{Compliance with ethical standards}

Conflict of interest AS has nothing to disclose. YN reports grants from Ono, grants from Taiho. YT reports personal fees from Ono, personal fees from Bristol-Myers Squibb, personal fees from Chugai, personal fees from AstraZeneca, personal fees from MSD, grants from KOTAI Biotechnology. HK, HH, SK,NN, KT, TK, YY, SM, and KS have nothing to disclose. DK reports personal fees from Takeda, personal fees from Chugai, personal fees from Lilly, personal fees from Merck serono, personal fees from Taiho, personal fees from Sysmex. AK reports grants and personal fees from Taiho, grants from Ono, grants from $\mathrm{Su}-$ mitomo Dainippon, grants from MSD. YK reports grants and personal fees from Takeda, personal fees from Bayer, personal fees from Lilly, grants and personal fees from Taiho, personal fees from MSD, personal fees from Sanofi, grants from Astra Zeneca, grants from Daiichi Sankyo, grants from Incyte, grants from Ono, grants from Boehringer Ingelheim, grants from Chugai, grants from Amgen, grants from Genmab. HT reports personal fees from Takeda, personal fees from Taiho, personal fees from Chugai, grants from Sysmex, grants from Daiichi Sankyo. TK reports grants and personal fees from MSD, grants from Astellas, grants from Bristol-Myers Squib, grants from Oncolys Biopharma, grants from Ono, grants from Shionogi. TD reports grants and personal fees from Abbie, personal fees from Amgen, personal fees from Bayer, grants and personal fees from Boeringer Ingelheim, grants and personal fees from Daiichi Sankyo, grants and personal fees from MSD, grants and personal fees from Novartis, personal fees from Sumitomo Dainippon, personal fees from Rakuten Medical, grants and personal fees from Taiho, personal fees from Takeda, personal fees from Astellas, grants and personal fees from Bristol-Myers Squibb, personal fees from Oncolys Biopharma, personal fees from Ono, grants from Eisai, grants from kyowa Hakko Kirin, grants from Lilly, grants from Merck Serono, grants from Novartis, grants from Pfizer, grants from Quintiles, grants from Sumitomo Group. TY reports grants and personal fees from Chugai, personal fees from Lilly, personal fees from Merck Serono, grants and personal fees from Sanofi, personal fees from Takeda, personal fees from Merck Biopharma, grants from GlaxoSmithKline, grants from MSD, grants from Nippon Boehringer Ingelheim, grants from Novartis, grants from Ono, grants from Dai- ichi Sankyo, grants from Parexel, grants from Sumitomo Dainippon. TY reports personal fees from Olympus, personal fees from Mejiseka Pharma, grants from SHIMADZU, grants from HOYA PENTAX, grants from Rakuten Medical. TK and TA has nothing to disclose. HN reports grants and personal fees from Ono, grants and personal fees from Bristol-Myers Squibb, grants and personal fees from Chugai, grants from Taiho, grants from Daiichi-Sankyo, grants from Kyowa Kirin, grants from Zenyaku Kogyo, grants from Astellas, grants from Sumitomo Dainippon, grants from Asahi-Kasei, grants from Astellas, grants from BD Japan. KS reports grants and personal fees from Astellas Phama, grants and personal fees from Lilly, personal fees from Bristol-Myers Squibb, personal fees from Takeda, personal fees from Pfizer, grants and personal fees from Ono Pharmaceutical, personal fees from Novartis, personal fees from Abbvie, personal fees from Yakult, grants from Dainippon Sumitomo Pharma, grants from Daiichi Sankyo, grants from Taiho Pharmaceutical, grants from Chugai Phaema, grants and personal fees from MSD, grants from Medi Science.

Ethics approval and consent to participate All procedures followed in this study were in accordance with the Declaration of Helsinki of 1964 and later versions and the Japanese Ethical Guidelines for Medical and Health Research Involving Human Subjects.

\section{References}

1. Tey J, Back MF, Shakespeare TP, Mukherjee RK, Lu JJ, Lee $\mathrm{KM}$, et al. The role of palliative radiation therapy in symptomatic locally advanced gastric cancer. Int J Radiat Oncol Biol Phys. 2007;67(2):385-8.

2. Hashimoto K, Mayahara H, Takashima A, Nakajima TE, Kato K, Hamaguchi T, et al. Palliative radiation therapy for hemorrhage of unresectable gastric cancer: a single institute experience. J Cancer Res Clin Oncol. 2009;135(8):1117-23.

3. Tey J, Choo BA, Leong CN, Loy EY, Wong LC, Lim K, et al. Clinical outcome of palliative radiotherapy for locally advanced symptomatic gastric cancer in the modern era. Medicine (Baltimore). 2014;93(22):e118.

4. Zou W, Wolchok JD, Chen L. PD-L1 (B7-H1) and PD-1 pathway blockade for cancer therapy: mechanisms, response biomarkers, and combinations. Sci Transl Med. 2016;8(328):328rv4.

5. Kang YK, Boku N, Satoh T, Ryu MH, Chao Y, Kato K, et al. Nivolumab in patients with advanced gastric or gastro-oesophageal junction cancer refractory to, or intolerant of, at least two previous chemotherapy regimens (ONO-4538-12, ATTRAC TION-2): a randomised, double-blind, placebo-controlled, phase 3 trial. Lancet. 2017;390(10111):2461-71.

6. Deng L, Liang H, Xu M, Yang X, Burnette B, Arina A, et al. STING-dependent cytosolic DNA sensing promotes radiationinduced type I interferon-dependent antitumor immunity in immunogenic tumors. Immunity. 2014;41(5):843-52.

7. Dovedi SJ, Adlard AL, Lipowska-Bhalla G, McKenna C, Jones $\mathrm{S}$, Cheadle EJ, et al. Acquired resistance to fractionated radiotherapy can be overcome by concurrent PD-L1 blockade. Cancer Res. 2014;74(19):5458-68.

8. Victor CT-S, Rech AJ, Maity A, Rengan R, Pauken KE, Stelekati E, et al. Radiation and dual checkpoint blockade activate non-redundant immune mechanisms in cancer. Nature. 2015;520(7547):373-7.

9. Nagasaka M, Zaki M, Kim H, Raza SN, Yoo G, Lin HS, et al. PD1/PD-L1 inhibition as a potential radiosensitizer in head and neck squamous cell carcinoma: a case report. J Immunother Cancer. 2016;4:83. 
10. Shi F, Wang X, Teng F, Kong L, Yu J. Abscopal effect of metastatic pancreatic cancer after local radiotherapy and granulocytemacrophage colony-stimulating factor therapy. Cancer Biol Ther. 2017;18(3):137-41.

11. Haymaker CL, Kim D, Uemura M, Vence LM, Phillip A, McQuail $\mathrm{N}$, et al. Metastatic melanoma patient had a complete response with clonal expansion after whole brain radiation and PD-1 blockade. Cancer Immunol Res. 2017;5(2):100-5.

12. Schoenhals JE, Seyedin SN, Tang C, Cortez MA, Niknam S, Tsouko E, et al. Preclinical rationale and clinical considerations for radiotherapy plus immunotherapy: going beyond local control. Cancer J. 2016;22(2):130-7.

13. Alomari AK, Cohen J, Vortmeyer AO, Chiang A, Gettinger S, Goldberg S, et al. Possible interaction of anti-PD-1 therapy with the effects of radiosurgery on brain metastases. Cancer Immunol Res. 2016;4(6):481-7.

14. Antonia SJ, Villegas A, Daniel D, Vicente D, Murakami S, Hui R, et al. Durvalumab after chemoradiotherapy in stage III non-smallcell lung cancer. N Engl J Med. 2017;377(20):1919-29.

15. Hui R, Ozguroglu M, Villegas A, Daniel D, Vicente D, Murakami $\mathrm{S}$, et al. Patient-reported outcomes with durvalumab after chemoradiotherapy in stage III, unresectable non-small-cell lung cancer (PACIFIC): a randomised, controlled, phase 3 study. Lancet Oncol. 2019;20:1670-80.

16. Eisenhauer EA, Therasse P, Bogaerts J, Schwartz LH, Sargent D, Ford R, et al. New response evaluation criteria in solid tumours: revised RECIST guideline (version 1.1). Eur J Cancer. 2009;45(2):228-47.

17. Lee SM, Kim SH, Lee JM, Im SA, Bang YJ, Kim WH, et al. Usefulness of CT volumetry for primary gastric lesions in predicting pathologic response to neoadjuvant chemotherapy in advanced gastric cancer. Abdom Imaging. 2009;34(4):430-40.

18. Association Japanese Gastric Cancer. Japanese classification of gastric carcinoma, 15th edn. Tokyo: Kanehara \& CO. Ltd.; 2017. (Japanese)

19. Kamada T, Togashi Y, Tay C, Ha D, Sasaki A, Nakamura Y, et al. PD-1(+) regulatory T cells amplified by PD-1 blockade promote hyperprogression of cancer. Proc Natl Acad Sci USA. 2019;116(20):9999-10008.

20. Tran DQ, Ramsey H, Shevach EM. Induction of FOXP3 expression in naive human CD4+FOXP3 T cells by T-cell receptor stimulation is transforming growth factor-beta dependent but does not confer a regulatory phenotype. Blood. 2007;110(8):2983-90.

21. Togashi Y, Nishikawa H. Regulatory T cells: molecular and cellular basis for immunoregulation. Curr Top Microbiol Immunol. 2017;410:3-27.

22. Saito T, Nishikawa H, Wada H, Nagano Y, Sugiyama D, Atarashi $\mathrm{K}$, et al. Two FOXP3(+)CD4(+) T cell subpopulations distinctly control the prognosis of colorectal cancers. Nat Med. 2016;22(6):679-84.
23. Lu K, He C, Guo N, Chan C, Ni K, Lan G, et al. Low-dose X-ray radiotherapy-radiodynamic therapy via nanoscale metal-organic frameworks enhances checkpoint blockade immunotherapy. Nat Biomed Eng. 2018;2(8):600-10.

24. Qin R, Olson A, Singh B, Thomas S, Wolf S, Bhavsar NA, et al. Safety and efficacy of radiation therapy in advanced melanoma patients treated with ipilimumab. Int J Radiat Oncol Biol Phys. 2016;96(1):72-7.

25. Young KH, Baird JR, Savage T, Cottam B, Friedman D, Bambina $\mathrm{S}$, et al. Optimizing timing of immunotherapy improves control of tumors by hypofractionated radiation therapy. PLoS ONE. 2016;11(6): e0157164.

26. Sundahl N, De Wolf K, Kruse V, Meireson A, Reynders D, Goetghebeur E, et al. Phase 1 dose escalation trial of ipilimumab and stereotactic body radiation therapy in metastatic melanoma. Int J Radiat Oncol Biol Phys. 2018;100(4):906-15.

27. Osa A, Uenami T, Koyama S, Fujimoto K, Okuzaki D, Takimoto $\mathrm{T}$, et al. Clinical implications of monitoring nivolumab immunokinetics in non-small cell lung cancer patients. JCI. Insight. 2018. https://doi.org/10.1172/jci.insight.59125.

28. Eriksson D, Stigbrand T. Radiation-induced cell death mechanisms. Tumour Biol. 2010;31(4):363-72.

29. Carvalho HA, Villar RC. Radiotherapy and immune response: the systemic effects of a local treatment. Clinics (Sao Paulo). 2018;73(suppl 1):e557s.

30. Deng L, Liang H, Fu S, Weichselbaum RR, Fu YX. From DNA damage to nucleic acid sensing: a strategy to enhance radiation therapy. Clin Cancer Res. 2016;22(1):20-5.

31. Liu C, Hu Q, Hu K, Su H, Shi F, Kong L, et al. Increased CD8+ $\mathrm{CD} 28+\mathrm{T}$ cells independently predict better early response to stereotactic ablative radiotherapy in patients with lung metastases from non-small cell lung cancer. J Transl Med. 2019;17(1):120.

32. Yang ZR, Zhao N, Meng J, Shi ZL, Li BX, Wu XW, et al. Peripheral lymphocyte subset variation predicts prostate cancer carbon ion radiotherapy outcomes. Oncotarget. 2016;7(18):26422-35.

33. Togashi Y, Shitara K, Nishikawa H. Regulatory T cells in cancer immunosuppression-implications for anticancer therapy. Nat Rev Clin Oncol. 2019;16(6):356-71.

34. Ayman OL, Shilpa B, David R, Lynn H, Raphael N, Eric C, Sana K. Regulatory $\mathrm{T}$ cells mediate resistance to radiotherapy in head and neck squamous cell carcinoma. J Clin Oncol. 2019;37(8_suppl):70.

Publisher's Note Springer Nature remains neutral with regard to jurisdictional claims in published maps and institutional affiliations.

\section{Affiliations}

\section{Akinori Sasaki ${ }^{1,2}$. Yoshiaki Nakamura ${ }^{1}$. Yosuke Togashi ${ }^{3}$. Hirofumi Kuno ${ }^{4}$. Hidehiro Hojo ${ }^{5}$. Shunichiro Kageyama ${ }^{5}$. Naoki Nakamura ${ }^{5} \cdot K_{\text {Kenji Takashima }}{ }^{6} \cdot$ Tomohiro Kadota $^{6} \cdot$ Yusuke Yoda $^{6} \cdot$ Saori Mishima $^{1} \cdot$ Kentaro Sawada $^{1}$. Daisuke Kotani ${ }^{1}$ - Akihito Kawazoe ${ }^{1}$. Yasutoshi Kuboki ${ }^{1}$. Hiroya Taniguchi ${ }^{1} \cdot$ Takashi Kojima $^{1} \cdot$ Toshihiko Doi $^{1}$. Takayuki Yoshino $^{1} \cdot$ Tomonori Yano $^{6} \cdot$ Tatsushi Kobayashi $^{4} \cdot$ Tetsuo Akimoto $^{2,5} \cdot$ Hiroyoshi Nishikawa $^{3} \cdot$ Kohei Shitara $^{1}$}

\author{
Akinori Sasaki \\ akisasak@east.ncc.go.jp \\ Yoshiaki Nakamura \\ yoshinak@east.ncc.go.jp
}

Yosuke Togashi

ytogashi1584@gmail.com

Hirofumi Kuno

hkuno@east.ncc.go.jp 
Hidehiro Hojo

hhojo@east.ncc.go.jp

Shunichiro Kageyama

skageyama@east.ncc.go.jp

Naoki Nakamura

naoknaka@east.ncc.go.jp

Kenji Takashima

ktakashi@east.ncc.go.jp

Tomohiro Kadota

tkadota@east.ncc.go.jp

Yusuke Yoda

yuyoda@east.ncc.go.jp

Saori Mishima

smishima@east.ncc.go.jp

Kentaro Sawada

kesawada@east.ncc.go.jp

Daisuke Kotani

dkotani@east.ncc.go.jp

Akihito Kawazoe

akawazoe@east.ncc.go.jp

Yasutoshi Kuboki

ykuboki@east.ncc.go.jp

Hiroya Taniguchi

hirtanig@east.ncc.go.jp

Takashi Kojima

takojima@east.ncc.go.jp

Toshihiko Doi

tdoi@east.ncc.go.jp
Takayuki Yoshino

tyoshino@east.ncc.go.jp

Tomonori Yano

toyano@east.ncc.go.jp

Tatsushi Kobayashi

takobaya@east.ncc.go.jp

Tetsuo Akimoto

takimoto@east.ncc.go.jp

Hiroyoshi Nishikawa

hnishika@ncc.go.jp

1 Department of Gastroenterology and Gastrointestinal Oncology, National Cancer Center Hospital East, 6-5-1 Kashiwanoha, Kashiwa, Chiba 277-8577, Japan

2 Courses of Advanced Clinical Research of Cancer, Juntendo University Graduate School of Medicine, Tokyo, Japan

3 Division of Cancer Immunology, Research Institute/Exploratory Oncology Research and Clinical Trial Centre, National Cancer Center Hospital East, Kashiwa, Japan

4 Department of Diagnostic Radiology, National Cancer Center Hospital East, Kashiwa, Japan

5 Division of Radiation Oncology and Particle Therapy, National Cancer Center Hospital East, Kashiwa, Japan

6 Department of Gastroenterology and Endoscopy, National Cancer Center Hospital East, Kashiwa, Japan 